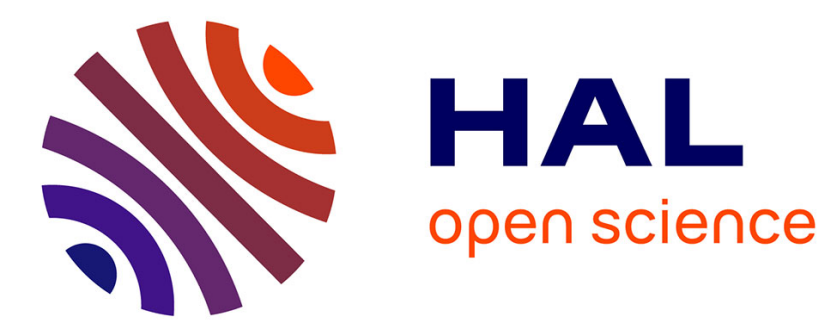

\title{
Optimization of micro-cavities for huge enhancement patterns in free-space and modal space
}

Paul Rouquette, Claude Amra, Myriam Zerrad, Michel Lequime

\section{To cite this version:}

Paul Rouquette, Claude Amra, Myriam Zerrad, Michel Lequime. Optimization of micro-cavities for huge enhancement patterns in free-space and modal space. Advances in Optical Thin Films VII, Sep 2021, Online Only, France. pp.3, 10.1117/12.2597032 . hal-03420168

\section{HAL Id: hal-03420168 https://hal.science/hal-03420168}

Submitted on 9 Nov 2021

HAL is a multi-disciplinary open access archive for the deposit and dissemination of scientific research documents, whether they are published or not. The documents may come from teaching and research institutions in France or abroad, or from public or private research centers.
L'archive ouverte pluridisciplinaire HAL, est destinée au dépôt et à la diffusion de documents scientifiques de niveau recherche, publiés ou non, émanant des établissements d'enseignement et de recherche français ou étrangers, des laboratoires publics ou privés. 


\title{
Optimization of micro-cavities for huge enhancement patterns in free- space and modal space
}

\author{
Paul Rouquette ${ }^{1,2}$, Claude Amra $^{1}$, Myriam Zerrad $^{1}$, Michel Lequime $^{1}$ \\ ${ }^{1}$ Aix Marseille Univ, CNRS, Centrale Marseille, Institut Fresnel, Marseille France \\ ${ }^{2}$ CILAS, Aubagne, France
}

\begin{abstract}
We show how to design huge enhancement of emission patterns in multilayer planar micro-cavities. Optimization is carried out arbitrarily for both the free-space pattern of plane waves and for the modal pattern of guided modes. The energy carried by these patterns is compared for various stacks, and their control is discussed.
\end{abstract}

Keywords: Micro-cavity, enhancement patterns, free-space, modal space.

\section{INTRODUCTION}

Optical sources within micro-cavities have been extensively studied for at least 2 decades [1]-[12]. These days it is well known that over-coating these cavities with multilayer optics allows their emission patterns in free-space to be modified. While sources within single cavities radiate a quasi-lambertian pattern, more elaborate structures (such as FabryPerot filters) enable the patterns in angular or spectral regions to be confined. Applications concern lighting and biophotonics, micro-sources and single photon sources, antennas, optical sensors, etc... The theoretical modelling has allowed to optimize the efficiency of the radiated pattern in free-space with remarkable results. The Fabry Perrot structure is, in most of the cases, used. However, the energy transferred to guided modes has been less studied because of the complexity of the problem.

In recent work we introduced the zero-admittance technique [16] for designing huge field enhancement in specific multilayer optics (ZAL coatings). This problem differs from that of micro-cavities since the optical coating is free of sources, and is illuminated from a high-index medium to allow total internal reflection (TIR) to occur. The field enhancement can be arbitrarily controlled (amplitude, wavelength, incidence and polarization, depth localization) with the design technique and increases with the layer number; its amplitude is bounded by intrinsic (absorption, roughness, thermal and non-linear effects, damage threshold) and extrinsic bandwidth phenomena such as divergence and spectral width, design accuracy and transverse uniformity) conditions. Here enhancement means that the electromagnetic stationary field in the coating is much greater (by several decades) than the incident field.

The objective of this paper is to show that similar techniques are available for designing huge enhancement of emission patterns in micro-cavities. Specific layers are introduced to over-coat given cavities and are first shown to create asymptotic poles in the reflection coefficient of the multilayer structure in the free-space (plane waves) frequency range. The result is a huge enhancement of the free-space pattern, by several decades. In a second step the technique is extended to the modal (guided waves) window, where another technique allows exact poles to be constructed and to attain huge enhancement of arbitrary modes. We compare the energy carried in free space to that transferred to the guided modes, and address the question of controlling this ratio. Results are discussed for a series of stacks. This technique could be of great interest in the fabrication of ultra-sensitive optical sensor and differs from other works that rely mostly on numerical optimization [13],[14].

\section{RECALLS ON MICRO-CAVITIES}

The aim of this section is to recall how the emission patterns in micro-cavities can be obtain. We work in a single wavelength (or harmonic) regime with a temporal dependence exp $(-j \omega t), \omega=2 \pi c / \lambda$ being the temporal pulsation, and $\lambda$ the illumination wavelength. All media are assumed to be linear, isotropic, and non-magnetic. The end media (substrate and superstrate) are assumed to be non-absorbing. The planar geometry of the all-dielectric stack is shown in Figure 1. Such a stack can be designed either as an optical filter or coating (when illuminated at infinity from the surrounding media), or a micro-cavity (when the sources are located within the stack) or a waveguide (case of integrated optics). In Figure 1 
all thicknesses have the same order of magnitude as the wavelength. At each interface (i) of the coating, an electric of magnetic current can be found and stands for the optical sources.

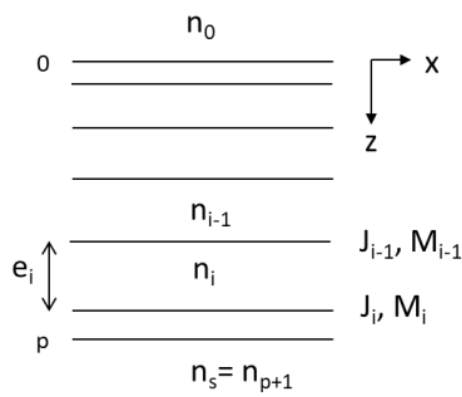

Figure 1: geometry of a planar multilayer stack, with $\mathrm{n}_{0}$ and $\mathrm{n}_{\mathrm{s}}$ the refractive indices of the superstrate and the substrate

Since we are interested in the emission pattern of optical sources in micro-cavities, it is useful to remind how the energy balance can be analysed in those structures. The energy balance can be written [3], [4], [15] as:

$$
F=\Phi+A
$$

where $F$ is the total power provided by the sources, $\Phi$ the Poynting flux through a closed surface surrounding the cavity, and $\mathrm{A}$ the absorption within the cavity. We assume the absence of interaction between fields and currents (weak regime), and that the cavity supports a unique electric current $\overrightarrow{J_{l}}(\vec{r})$ located at one interface (i). Under these conditions, in a single wavelength regime each term of relation (1) follows [3], [4], [15]:

$$
\frac{d F}{d \varphi}=\int_{0}^{+\infty} f(\vec{\sigma}) d \sigma \quad \frac{d \Phi}{d \varphi}=\int_{0}^{+\infty} g(\vec{\sigma}) d \sigma \quad A=\frac{\omega}{2} \int_{0}^{z_{p}} \mathfrak{J}\{\tilde{\epsilon}(z)\}|E(\vec{\sigma}, z)|^{2} d z
$$

where $\vec{\sigma}=\sigma(\cos \varphi, \sin \varphi)$ is the spatial pulsation in polar direction $\varphi$ in the range $[0 ; 2 \pi], \vec{r}=(x, y)$ the spatial transverse coordinates and $z$ an altitude in a direction perpendicular to the multilayer. Note that all energy quantities are given per unit of current surface area. The stationary electromagnetic field in the volume of the stack is denoted $E(z, \sigma)$. Also, recall that all quantities depend on $\omega$, whose notation is omitted for the sake of simplicity. The temporal Fourier transform of permittivity $\varepsilon(t, z)$ is denoted $\tilde{\varepsilon}(z)$.

In relation (2) the $f$ and $g$ functions are spectral densities of power and flux respectively, per unit spatial pulsation and polar angle. The power density is given as [3], [4], [15]:

$$
f(\vec{\sigma})=\frac{d F}{d \sigma d \varphi}=2 \pi^{2} \Re\left\{\frac{1}{\Delta \mathrm{Y}_{\mathrm{i}}(\sigma)}\right\}\left|\hat{J}_{i}(\vec{\sigma})\right|^{2}
$$

where $\hat{J}_{i}(\sigma)$ is the spatial Fourier transform of $\vec{J}_{l}(\vec{r})$ and the quantity $\Delta Y_{i}=Y_{i}^{\prime}-Y_{i}$ is the difference in the complex admittances from each side of surface (i), that is, in layers (i) and (i-1) respectively. Note that the admittances are discontinuous at surface $i$ where the current is present. Relation (3) shows how the cavity coefficient $\frac{1}{\Delta \mathrm{Y}_{\mathrm{i}}(\sigma)}$ drives the power provided by the current, which can be inhibited or enhanced (at constant current). It should be stressed that all terms in relation (1) are cavity-related (they depend on the cavity coefficient), so that the optical balance is always satisfied [15].

The flux density emerging into the end media is given by:

$$
g(\vec{\sigma})=g^{-}(\vec{\sigma})+g^{+}(\vec{\sigma})
$$

Where $g^{ \pm}$is relative to the flux by reflection $\left(g^{-}\right)$or transmission $\left(g^{+}\right)$, that is:

$$
g^{-}(\vec{\sigma})=\frac{\mathrm{d} \Phi^{-}}{\operatorname{d} \sigma \mathrm{d} \varphi}=2 \pi^{2} \sigma \Re\left\{\tilde{n}_{0}\right\}\left|E_{0}^{-}(\vec{\sigma})\right|^{2} \text { and } g^{+}(\vec{\sigma})=\frac{\mathrm{d} \Phi^{+}}{\operatorname{d} \sigma \mathrm{d} \varphi}=2 \pi^{2} \sigma \Re\left\{\tilde{n}_{S}\right\}\left|E_{S}^{-}(\vec{\sigma})\right|^{2}
$$

In relations (5), $\tilde{n}$ denotes the effective index in the superstrate $\left(\tilde{n}_{0}\right)$ or in the substrate $\left(\tilde{n}_{s}\right)$. 
Throughout all these formulae, light polarization is considered by the effective indices and admittances. Finally, one can check [15] for a lossless cavity that the provided power is equal to the sum of the emerging fluxes.

In relation (5) the flux is given as a function of fields, while in (3) the power was given as a function of the current. We will also need to express the power as a function of the fields [15] which can be written in the absence of magnetic currents:

$$
f(\vec{\sigma})=f^{-}(\vec{\sigma})+f^{+}(\vec{\sigma})=2 \pi^{2} \sigma\left[\mathfrak{R}\left\{Y_{i}^{\prime}\right\}\left|E_{i}^{\prime}(\sigma)\right|^{2}-\mathfrak{R}\left\{Y_{i}\right\}\left|E_{i}(\sigma)\right|^{2}\right]=2 \pi^{2} \sigma \Re\left\{\Delta Y_{i}\right\}\left|E_{i}(\sigma)\right|^{2}
$$

As an illustration and because of future need for normalization in the next sections, the emission pattern of 2 single cavities is plotted at a wavelength of $633 \mathrm{~nm}$ in Figure 2. The first cavity (on the left) is a single very thin (1nm) high-index layer between an air superstrate and an air substrate, with the current located at half distance from each interface. We observe a quasi-lambertian pattern in both reflection and transmission, which will be used for normalization of the patterns from other cavities. The second cavity (on the right) is a $Q W=L(H L)^{q}$ stack matched at wavelength $\lambda_{0}^{0}=633 \mathrm{~nm}$ for normal incidence $\theta_{0}^{0}=0^{\circ}$. The current is located at the top surface of the mirror. This second cavity pattern is normalized at each angle by the previous one, which highlights the cavity effect (moderate enhancement in reflection by a factor of 4 , and strong reduction in transmission). TE polarization is used for all patterns. Notice that huge enhancement is not expected here and will be discussed in the next section.
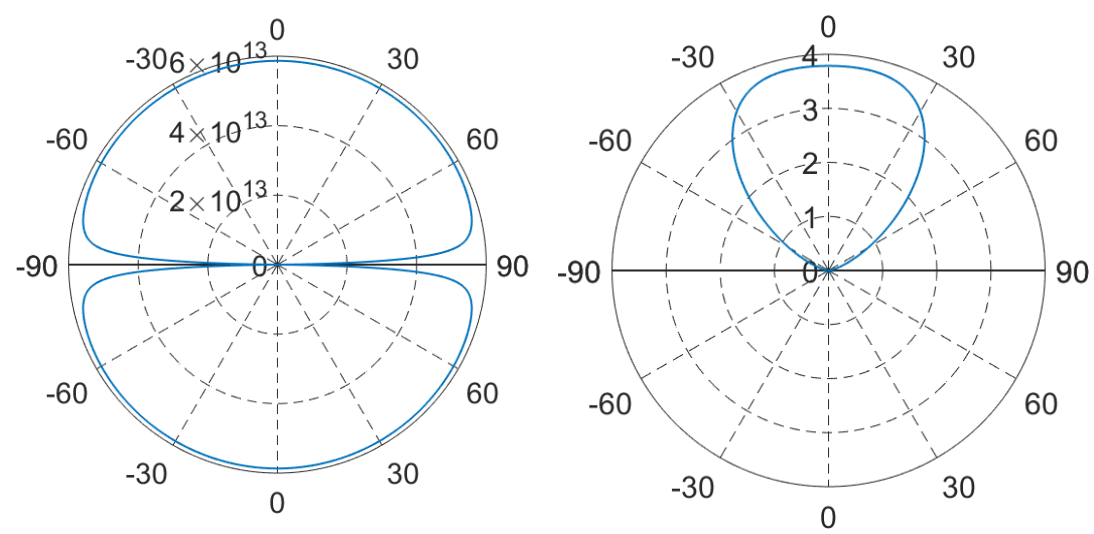

Figure 2: Angular patterns at $633 \mathrm{~nm}$ for a single very thin layer cavity (left side, SI units) and for a quarter-wave stack (right side). The second pattern is normalized with respect to the first (see text). TE polarization.

\section{ENHANCEMENT PATTERNS IN FREE SPACE WITH ZAL COATINGS}

\subsection{Analytical discussion}

Field enhancement still remains one of the key objectives of nanophotonics [17],[18], regardless of the geometry (planar, radial, concentric) of the component. Enhancement means that the stationary field in the stack is much greater than the input (incident) collimated (plane wave) field in the superstrate. In the case of planar multilayers, we recently highlighted an analytical design technique for creating huge (several decades) field enhancement with zero-admittance layers [15], [16] a technique that is relevant for threshold reduction of micro-sources and high-sensitivity optical sensing. The enhancement can be controlled in amplitude and for arbitrary illumination conditions (incidence, wavelength and polarization), provided the stack is under total internal reflection (TIR). Hence the superstrate is a high-index material with respect to the substrate material $\left(n_{0}>n_{s}\right)$ and oblique incidence $\left(\theta_{0}\right)$ in the superstrate is required beyond the refractionlimit angle, that is, in terms of spatial pulsation:

$$
\sigma_{0}=k_{0} \sin \theta_{0}>k_{S} \text { with } k_{0, S}=2 \pi n_{0, S} / \lambda \text { and } \sigma_{0} \text { is the incident spatial pulsation }
$$

The TIR regime is a key feature since it allows the complex admittance to be cancelled at a particular altitude $z_{c}$ of the stack (which is the origin of huge enhancement in quarter-wave stacks under TIR), while in free space this zero admittance value must be approached asymptotically (as in Fabry-Perot filters). So, if one considers a quarter wave stack with a ZAL layer over coating the stack we obtain, at a specific spatial pulsation $\sigma_{c}>k_{S}$ and at the top interface of the ZAL layer $(z=$ $\left.z_{p-1}\right)$ :

$$
Y^{\prime}\left(\sigma_{c}, z=z_{p-1}\right)=0
$$


We are now interested in the optimization of light emitted by the micro-cavity in the absence of illumination. As per relation (3), maximum enhancement in the cavity would be provided at spatial frequencies $\sigma_{m}$ which satisfy the condition:

$$
\Delta Y_{i}\left(\sigma_{m}\right)=\Delta Y_{i}^{\prime}\left(\sigma_{m}, z=z_{i}\right)=0
$$

Note that relations (8-9) are respectively related to TIR $\left(k_{s}<\sigma_{c}<k_{0}\right)$ and modal $\left(\max \left(k_{0}, k_{s}\right)<\sigma_{m}<\max \left(k_{i}\right)\right)$ frequency ranges which do not intersect [4], [15] so that they do not seem related a priori. This result is illustrated in Figure 3 , where the abscissa is the normalized spatial frequency $\left(v^{*}=\lambda \sigma / 2 \pi\right)$ analogous to $n \sin \theta$.

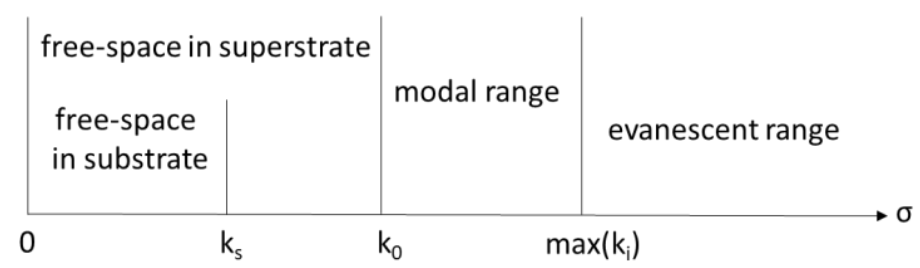

Figure 3: Free-space and modal frequency windows. The abscissa is the normalized spatial frequency

Since we are concerned with an optimization of the reflected pattern only, we start with relation (6) which can be further developed as [15]:

$$
f^{-}(\vec{\sigma})=-2 \pi^{2} \sigma \mathfrak{R}\left\{Y_{i}\right\} \frac{\left|\hat{J}_{i}(\vec{\sigma})\right|^{2}}{\left|\Delta Y_{i}\right|^{2}}
$$

We assume the current to be located at the top interface (p-1) of the ZAL layer. With relation (8), we obtain:

$$
\Delta Y\left(\sigma_{c}, z_{p-1}\right)=-Y\left(\sigma_{c}, z_{p-1}\right)=-Y_{p-1}\left(\sigma_{c}\right)
$$

So that:

$$
f^{-}\left(\overrightarrow{\sigma_{c}}\right)=2 \pi^{2} \sigma_{c} \Re\left\{Y_{p-1}\left(\sigma_{c}\right)\right\} \frac{\left|\hat{J}_{i}\left(\sigma_{c}\right)\right|^{2}}{\left|\mathrm{Y}_{\mathrm{p}-1}\left(\sigma_{c}\right)\right|^{2}}
$$

and the question is whether this last quantity can be maximized at $\sigma_{c}$. For that we use the transfer matrix from interface 0 to $\mathrm{p}-1$ to find an expression of $\mathrm{Y}_{\mathrm{p}-1}[15]$ :

$$
Y_{p-1}\left(\sigma_{c}\right)=\frac{\tilde{n}_{L}^{2}}{\tilde{n}_{0}}\left(\frac{1}{\beta^{2 q}}\right) \text { with } \beta=\tilde{n}_{H} / \tilde{n}_{L}
$$

We can then conclude that the single wavelength cavity pattern at the ZAL frequency $\sigma_{c}<k_{0}$ is enhanced in the form:

$$
f^{-}\left(\overrightarrow{\sigma_{c}}\right)=2 \pi^{2} \sigma_{c} \beta^{2 q} \frac{\tilde{n}_{0}^{2}}{\tilde{n}_{L}}\left|\hat{J}_{i}\left(\sigma_{c}\right)\right|^{2}
$$

The key result here is the proportionality to the term $\beta^{2 q}$ of the effective index ratio, which creates a huge enhancement pattern of the micro-cavity.

It should be stressed that huge enhancement occurred at the ZAL frequency $\sigma_{c}$ though this frequency does not belong to the modal frequency, in contrast with the poles $\sigma_{m}$. The reason is that $\sigma_{c}$ is not an exact, but an asymptotic zero of the admittance difference:

$$
\lim _{q \rightarrow \infty} \Delta Y_{p-1}\left(\sigma_{c}\right)=0
$$

\subsection{Numerical results}

In Figure 4 we have plotted the angular pattern of a multilayer stack with a ZAL layer. The design of the coating is Glass/L(HL) $/$ ZAL/Air. The stack was formerly designed for a free-space illumination for TE polarization, at wavelength $\lambda_{0}^{0}=633 \mathrm{~nm}$ and incidence $\theta_{0}^{0}=45^{\circ}$. Here there is no free-space illumination, but a current located at the top surface of the ZAL layer (interface $\mathrm{p}-1=13$ with $\mathrm{q}=6$ ). The emission pattern is plotted for TE polarization in the incidence plane 
$\left(\varphi=0^{\circ}\right)$ at wavelength $\lambda_{0}=\lambda_{0}^{0}$. In order to quantify the gain of this cavity, the pattern is normalized at each normal angle $\theta$ to the pattern value (at the same angle) of the thin layer cavity to the left of Figure 2.

Following (14) the cavity is expected to enhance the source emission at the same $\lambda_{0}^{0}$ and $\theta_{0}^{0}$. This is indeed what we observe in Figure 4 since there is an intense peak of four decades enhancement at $45^{\circ}$. Note also the narrow angular width $\Delta \theta_{0}$ of the peak, where most of the energy is concentrated; the percentage of energy in the peak is $14 \%$ for $\Delta \theta_{0}=0.01^{\circ}$ and $36 \%$ for $\Delta \theta_{0}=0.1^{\circ}$. These values must be multiplied by a factor of 2 because of the symmetrical peak. Contrary to its integral, the peak magnitude is not bounded due to the fact that the width and amplitude of the peaks are respectively proportional and inversely proportional to the imaginary indices of the thin film materials. Other peaks are present but cannot be seen in the left-hand figure because their amplitude is much lower by several decades. They can be seen on the right-hand figure where $\mathrm{I}_{0}$ is plotted for the reflected pattern and $\mathrm{I}_{\mathrm{S}}$ for the transmitted pattern.
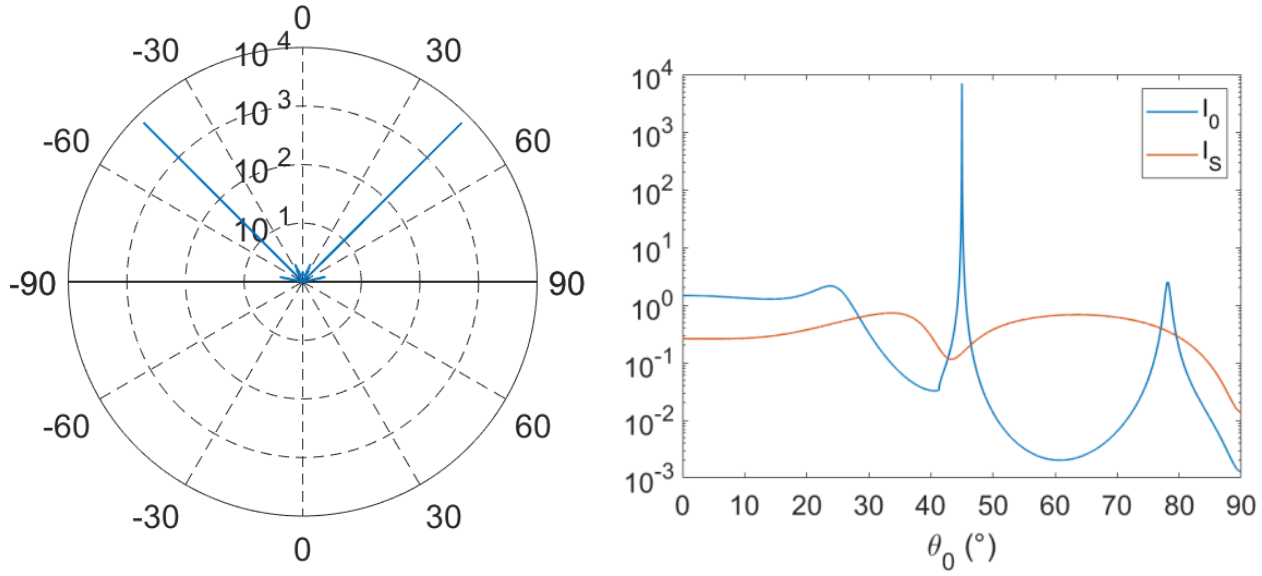

Figure 4: TE normalized angular pattern at $633 \mathrm{~nm}$ of the ZAL micro-cavity in polar (left figure) and cartesian (right figure) coordinates (see text).

\section{ENHANCEMENT OF GUIDED WAVE IN MICRO-CAVITIES}

Until this point, we were limited to free-space optics and never considered the energy carried by guided modes within the cavity in directions parallel to the interfaces. In this section we address the question of modal efficiency, that is, the ability to reach a maximum power carried by these modes without radiative losses. In other words, starting from a given source in the cavity, the question is how to optimize the power provided to the modal window, or to a specific mode in this window, and to quantify this power in comparison with the free-space power in the surrounding media.

\subsection{Working with the ZAL coating and the ZAL frequency}

An initial idea was to keep the same ZAL coating but modify the superstrate so that the zero-admittance frequency $\sigma_{c}$ is transferred to the modal space. Hence the glass superstrate is replaced by air, which turns the modal frequency range into:

$$
\max \left(k_{S}, k_{S}\right)=k_{S}<\sigma_{c}<\max \left(k_{i}\right)=k_{H}
$$

where $\mathrm{k}_{S}$ is for the air medium. The surface current is still located at the top surface (p-1) of the ZAL layer. The power density $f(\sigma, \varphi=0)$ of the cavity is shown in Figure 5 for TE polarization. Eight modes can be seen in the modal window, with the $\mathrm{TE}_{0}$ mode at the highest frequency, while the zero-admittance frequency $\sigma_{c}$ is close to the $\mathrm{TE}_{6}$ mode (with the highest amplitude). Actually, the $\mathrm{TE}_{6}$ frequency tends to the ZAL frequency when the layer number of the QW stack increases, that is:

$$
\lim _{q \rightarrow \infty} \sigma_{6}=\sigma_{m}
$$

As expected, we observe an intense peak in the vicinity of the ZAL frequency $\sigma_{c} \approx \sigma_{6}$. 


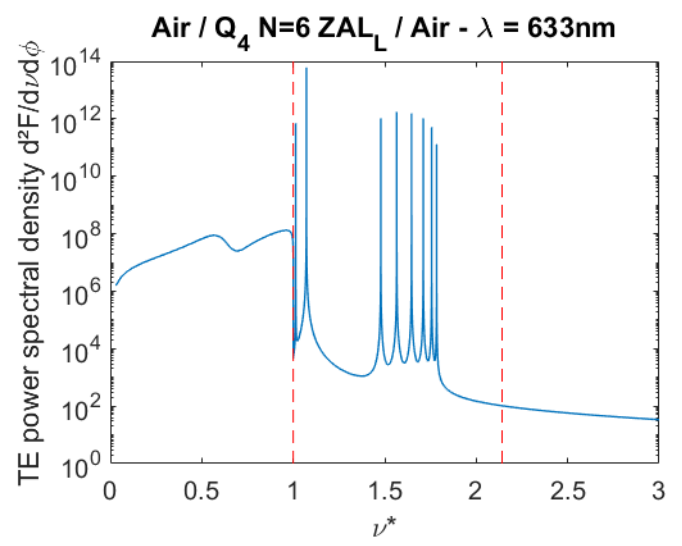

Figure 5: Power density versus normalized frequency, plotted for the ZAL cavity where the superstrate is replaced by air

Recall here that in contrast with the free-space situation where the poles are complex, there are 8 exact poles in the modal window at which the reflection function diverges. For that reason we used small imaginary indices $\left(10^{-6}\right)$ for thin film dielectric materials in order to remove these singularities.

Results can be found in Table 1. In this table we give the modal energy carried by each mode and for different current localization after normalization by the energy provided in the free-space window. This allows the efficiency of the process to be quantified. We first observe that, when the current is localized on the p-1 interface (\#13), the total modal power (last column) is greater by a factor of 4 than the free-space power, and that among the modes the greatest energy is carried by the $\mathrm{TE}_{6}$ mode (close to the ZAL frequency). Results vary with the depth localization of current. The greatest modal efficiency is reached at the $9^{\text {th }}$ interface, with maximum power transferred to the $\mathrm{TE}_{4}$ mode.

\begin{tabular}{|c|c|c|c|c|c|c|c|c|c|}
\hline Modes & $\mathrm{TE}_{0}$ & $\mathrm{TE}_{1}$ & $\mathrm{TE}_{2}$ & $\mathrm{TE}_{3}$ & $\mathrm{TE}_{4}$ & $\mathrm{TE} 5$ & $\mathrm{TE} 6$ & $\mathrm{TE}_{7}$ & total \\
\hline Inter 0 & 0.0040 & 0.0154 & 0.0323 & 0.0504 & 0.0596 & 0.0390 & 0.0137 & 1.2431 & 1.4576 \\
\hline Inter 1 & 0.4103 & 1.4034 & 2.3922 & 2.7552 & 2.1810 & 0.8954 & 0.0065 & 0.0177 & 10.0617 \\
\hline Inter 2 & 0.3701 & 1.1269 & 1.5607 & 1.2986 & 0.6286 & 0.1265 & 0.0085 & 0.8513 & 5.9712 \\
\hline Inter 3 & 0.9758 & 1.7215 & 0.5457 & 0.1399 & 1.6293 & 1.5430 & 0.0138 & 0.0030 & 6.5720 \\
\hline Inter 4 & 1.5858 & 2.1256 & 0.1889 & 0.9196 & 2.6219 & 1.2934 & 0.0041 & 0.4536 & 9.1929 \\
\hline Inter 5 & 1.3600 & 0.4179 & 0.6843 & 1.2168 & 0.0979 & 1.7770 & 0.0374 & 0.0003 & 5.5917 \\
\hline Inter 6 & 1.8752 & 0.2240 & 1.4767 & 0.8784 & 0.7534 & 2.0425 & 0.0011 & 0.1258 & 7.3772 \\
\hline Inter 7 & 1.7853 & 0.1886 & 1.4491 & 0.7620 & 0.7940 & 1.8889 & 0.1490 & 0.0002 & 7.0173 \\
\hline Inter 8 & 1.3741 & 0.3922 & 0.7328 & 1.1729 & 0.1321 & 1.7746 & 0.0003 & 0.0322 & 5.6113 \\
\hline Inter 9 & 1.9440 & 2.5291 & 0.1831 & 1.2213 & 3.1648 & 1.4529 & 0.7466 & 0.0040 & 11.2458 \\
\hline Inter 10 & 0.9069 & 1.5617 & 0.4534 & 0.1629 & 1.5353 & 1.3530 & 0.0001 & 0.0109 & 5.9843 \\
\hline Inter 11 & 0.6583 & 1.9798 & 2.6793 & 2.1423 & 0.9655 & 0.1687 & 2.1841 & 0.0149 & 10.793 \\
\hline Inter 12 & 0.4006 & 1.3564 & 2.2710 & 2.5431 & 1.9307 & 0.7490 & $4 * 10^{-5}$ & 0.0044 & 9.2552 \\
\hline Inter 13 & 0.0093 & 0.0359 & 0.0752 & 0.1172 & 0.1390 & 0.0917 & 3.6326 & 0.0249 & 4.1258 \\
\hline Inter 14 & 0.0052 & 0.0205 & 0.0446 & 0.0736 & 0.0939 & 0.0673 & 4.3954 & 0.0353 & 4.7357 \\
\hline
\end{tabular}

Table 1: Energy provided to each mode, after normalization by the energy provided in the free-space window. ZAL cavity with air superstrate and substrate. TE polarization. Each line is for a particular current localization (see text)

\subsection{Designing an exact pole with a MZAL coating}

In sub-section 3.1 we worked with the ZAL coating and observed that the zero-admittance (ZAL) frequency $\sigma_{c}$ was an asymptotic pole (see relation (15)). The best optimization would be to design an arbitrary exact pole (as opposed to asymptotic pole). While this was not possible in free space where exact poles are necessarily complex [10], the modal window offers the opportunity to construct an exact pole. To this end, we consider a QW stack alone and, in the absence of absorption, look for real pole $\sigma_{m}$ that satisfies:

$$
\Delta Y_{p}\left(\sigma_{m}\right)=0 \Leftrightarrow Y_{p}\left(\sigma_{m}\right)=\tilde{n}_{S}\left(\sigma_{m}\right)
$$


We coat the QW stack with a with a single layer of refractive index $\mathrm{n}$ and we seek the thickness layer that satisfies the condition $Y_{p+1}=\tilde{n}_{S}$, where subscript $(\mathrm{p}+1)$ is used to localize the new last interface of the stack. The admittances $Y_{p+1}$ and $Y_{p}$ are related through:

$$
Y_{p+1}=\frac{Y_{p} \cos \delta+j \tilde{n} \sin \delta}{\cos \delta+j \frac{Y_{p}}{\tilde{n}} \sin \delta} \quad \text { with } \quad \delta=\sqrt{k^{2}-\sigma^{2}} e
$$

Due to the lossless regime of the guided waves, the 2 emerging effective indices $\left(\tilde{n}_{0}\right.$ and $\left.\tilde{n}_{s}\right)$ and the 2 admittances are pure imaginary numbers $\left(Y=j Y^{\prime \prime}\right)$ [3], [4], [15] while the effective indices in the layer media are real $\left(\mathrm{k}_{s}<\sigma_{m}<\mathrm{k}_{L}<\right.$ $\left.\mathrm{k}_{H}\right)$. Seeking a solution for $Y_{p+1}\left(\sigma_{m}\right)=\tilde{n}_{S}\left(\sigma_{m}\right)$, we obtain:

$$
\tan \delta=\tilde{n} \frac{\tilde{n}_{S}^{\prime \prime}-Y_{p}^{\prime \prime}}{\tilde{n}^{2}+\tilde{n}_{S}^{\prime \prime} Y_{p}^{\prime \prime}}
$$

Relation (20) gives the thickness of the additional layer once its refractive index is chosen. By analogy with the ZAL layer, such a layer will be denoted MZAL to refer to the modal window to which it applies. Note that while the ZAL layer cancels the admittance in the free-space window, the MZAL layer cancels an admittance difference in the modal window.

Note that a MZAL could also be designed at the top interface. For that, following the same method, we coat the stack with a single layer of refractive index $\mathrm{n}$ and we seek the thickness that satisfies the condition $Y_{-1}^{\prime}=-\tilde{n}_{0}$, where the subscript $(-1)$ is used to localize the new top interface of the stack. We obtain:

$$
\tan \delta=\tilde{n} \frac{\tilde{n}_{0}^{\prime \prime}+Y_{0}^{\prime \prime}}{\tilde{n}^{2}-\tilde{n}_{0}^{\prime \prime} Y_{0}^{\prime \prime}}
$$

The MZAL layer allows an exact pole to be constructed whatever the coating design. Hence a complex stack is not required, and for this reason we address the case of a single layer over-coated with a MZAL layer on the superstrate side. The design of the coating is Air/MZAL H/Air and the question is how to quantify its performance with respect to the previous coatings. The MZAL is a high-index layer still calculated for a pole at $45^{\circ}$ and $633 \mathrm{~nm}$ wavelength. Results are plotted in Figure 6 for 3 different current localizations. Two poles appear in the modal window for TE polarization. The modal efficiency is given in Table 2. A maximum value is obtained at the top surface of the single layer (\#1), with maximum energy transferred to the $\mathrm{TE}_{0}$ mode that we constructed. We also notice that the modal efficiency is much greater (19) than that (4) of the QW stack, which highlights the results of this sub-section. We have checked that such efficiency can be increased (up to 25fold) if the high index material $\left(\mathrm{Ta}_{2} \mathrm{O}_{5}\right)$ is replaced by a higher index one $\left(\mathrm{Nb}_{2} \mathrm{O}_{5}, \mathrm{n}=2.3216\right.$ at $\left.633 \mathrm{~nm}\right)$ as is currently used in thin films. Note, however, that though these single stacks provide high efficiency in the modal window on account of their exact poles, this efficiency vanishes in free space since their behaviour is not asymptotic.

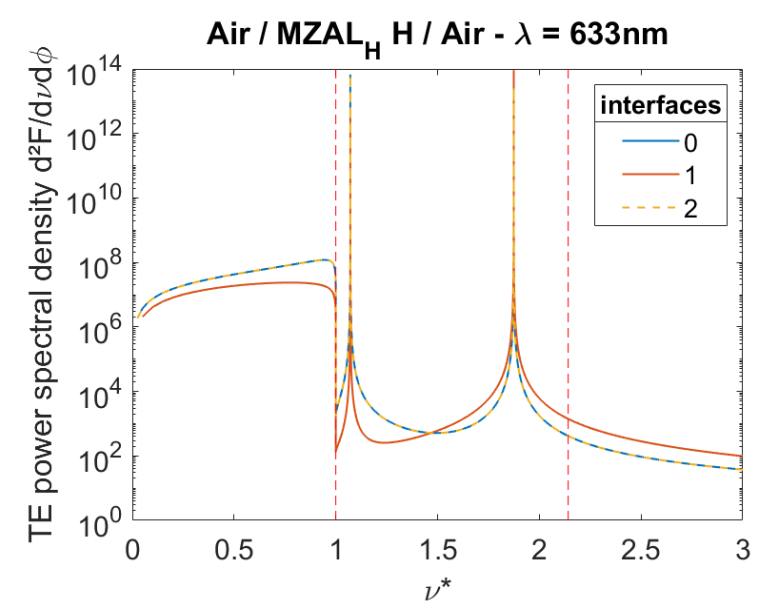

Figure 6 : power density for a single layer coated with a MZAL layer (see text) 


\begin{tabular}{|c|c|c|c|}
\hline Modes & $\mathrm{TE}_{0}$ & $\mathrm{TE}_{1}$ & total \\
\hline Inter 0 & 2.5355 & 1.7743 & 4.3098 \\
\hline Inter 1 & 18.7449 & 0.3490 & 19.0940 \\
\hline Inter 2 & 2.5355 & 1.7743 & 4.3098 \\
\hline
\end{tabular}

Table 2: Energy provided to each mode, after normalization by the energy provided in the free-space window. Case of a single layer coated with a MZAL layer. TE polarization. Each line is for a particular surface localization (see text)

\section{REFERENCES}

[1] H. Rigneault, S. Monneret, "Modal analysis of spontaneous emission in a planar micro-cavity," Phys. Rev. A 54, 2356-2368 (1996).

[2] H. Rigneault, S. Maure, and C. Amra, "Spontaneous emission in multilayer microcavities: modal theory extended with Fourier-Green analysis for dissipative structures," Pure and Applied Optics 7, 549-563 (1998).

[3] Claude Amra and Sophie Maure, "Electromagnetic power provided by sources within multilayer optics: free-space and modal patterns," J. Opt. Soc. Am. A 14, 3102-3113 (1997).

[4] Claude Amra and Sophie Maure, "Mutual coherence and conical pattern of sources optimally excited within multilayer optics," J. Opt. Soc. Am. A 14, 3114-3124 (1997).

[5] H. Benisty, H. De Neve, and C. Weisbuch, "Impact of Planar Microcavity Effects on Light Extraction-Part I: Basic Concepts and Analytical Trends", IEEE Journal of Quantum Electronics 34, (1998).

[6] H. Benisty, H. De Neve, and C. Weisbuch, "Impact of Planar Microcavity Effects on Light Extraction-Part II: Selected Exact Simulations and Role of Photon Recycling", IEEE Journal of Quantum Electronics 34, (1998).

[7] H. Benisty, R. Stanley and M. Mayer, "Method of source terms for dipole emission modification in modes of arbitrary planar structures", J. Opt. Soc. Am. A 15 (1998).

[8] G. Björk, S. Machida, Y. Yamamoto and K. Igeta, "Modification of spontaneous emission rate in planar dielectric microcavity structures", Physical Review A 44 (1991).

[9] D. Delbeke, R. Bockstaele, P. Bienstman, R. Baets and H. Benisty, "High-efficiency semiconductor resonant-cavity light-emitting diodes: a review," IEEE Journal of Selected Topics in Quantum Electronics 8, 189-206 (2002).

[10] D. G. Deppe and C. Lei, "Spontaneous emission from a dipole in a semiconductor microcavity”, Journal of Applied Physics 70, 3443 (1991).

[11]L'uboš Polerecký, Jaroslav Hamrle, and Brian D. MacCraith, "Theory of the radiation of dipoles placed within a multilayer system," Appl. Opt. 39, 3968-3977 (2000).

[12]H. Riel, S. Karg, T. Beierlein, W. Rieß and K. Neyts, "Tuning the emission characteristics of top-emitting organic light-emitting devices by means of a dielectric capping layer: An experimental and theoretical study", Journal of Applied Physics 94, 5290 (2003).

[13] P. Munzert, N. Danz, A. Sinibaldi, F. Michelotti, "Multilayer coatings for Bloch surface wave optical biosensors", Surface \& Coatings Technology 314, 79-84 (2017).

[14]F. Michelotti, R. Rizzo, A. Sinibaldi, P. Munzert, C. Wächter, and N. Danz, "Design rules for combined label-free and fluorescence Bloch surface wave biosensors," Opt. Lett. 42, 2798-2801 (2017).

[15] C. Amra, M. Lequime, and M. Zerrad, [Electromagnetic Optics of Thin-Film Coatings: Light Scattering, Giant Field Enhancement, and Planar Microcavities], Cambridge University Press (2021).

[16] C. Amra, M. Zerrad, F. Lemarchand, A. Lereu, A. Passian, J. Antonio Zapien, and M. Lequime, "Energy density engineering via zero admittance domains in all-dielectric stratified materials," Phys. Rev. A 97, 023819 (2018).

[17] Bahaa E. A. Saleh and Malvin C. Teich, [Fundamentals of Photonics], $3^{\text {rd }}$ edition, Wiley (2019).

[18] Lukas Novotny, Bert Hecht, [Principles of Nano-Optics], $2^{\text {nd }}$ edition, Cambridge University Press (2012). 